\author{
Military Technical College \\ Kobry El-Kobbah, \\ Cairo, Egypt
}

\author{
$14^{\text {th }}$ International Conference on \\ Applied Mechanics and \\ Mechanical Engineering.
}

\title{
The Model of Micro-Fluidic Pump with Vibrating Boundaries
}

\section{By}

\author{
Nihad E. Daidzic*
}

MD Shakhawath Hossain**

\begin{abstract}
:
A mathematical model and numerical simulations of the new shear-driven micro-fluidic pump concept is presented. The flow of the Newtonian and shear-thinning non-Newtonian fluid in plane 2D geometry, micro-channel, or pipe is achieved by oscillatory motion of the channel walls. The oscillatory flow for finite $2 \mathrm{D}$ geometry and ramp quasi-periodic boundary conditions is presented resulting in positive flow rate for Newtonian and some rheological fluids. Various layouts of the oscillatory motive plates can be achieved. Such micro-fluidic pumps can be easily arranged in a serial or parallel layout to deliver desired flow rates and/or efforts. Proposed shear-driven micro-fluidic pump can find variety of applications in supporting blood flow in vascular channels, MEMS fluidic systems, nanotechnology, food processing, automotive industry, etc.
\end{abstract}

\section{Keywords:}

Micro-fluidic pump, MEMS, Stokes $2^{\text {nd }}$ problem, Oscillatory flow, Fourier decomposition, Numerical finite-difference method, Boundary layer. 
* Aviation Department, Minnesota State University, Mankato, MN 56001, USA

** Mech. Eng. Department, Minnesota State University, Mankato, MN 56001, USA

\section{Introduction:}

A large number of scientific literatures have been devoted to the topic of the Stokes' $2^{\text {nd }}$ problem. A classical Stokes' $2^{\text {nd }}$ problem represents one of the very few known exact solutions of the Navier-Stokes (NS) equations.

A harmonically oscillating infinite flat plate sets the semi-infinite fluid in motion, which after the initial transients dies out settles into steady-state oscillatory flow. The exact solutions of the classical Stokes's $2^{\text {nd }}$ problem can be found, for e.g., in books by Lamb [1], Schlichting [2], and Batchelor [3]. This problem is very similar to the celebrated Kelvin's problem of penetration of harmonically induced temperature waves in a semi-infinite medium as shown in classical text by Carslaw and Jaeger [4].

In recent years, the topic of Stokes' $2^{\text {nd }}$ problem regained attention due to the possible application in MEMS, nanotechnology, etc. Article by Panton [5], and more recently by Erdogan [6], Khaled and Vafai [7], and Muzychka et al. [8], was discussing various analytical solutions of the Stokes' $1^{\text {st }}$ and $2^{\text {nd }}$ problem and Couette flow in general. These solutions were mostly concerned with the transient and steady-state velocity distribution of a Newtonian fluid in a semi-infinite domain, with and without slip condition and for various harmonic excitations. Ai and Vafai [9] numerically solved nonlinear Stokes's $2^{\text {nd }}$ problem using several models of non-Newtonian (rheological) fluids.

Recently, a micro-axial blood pump model was presented by Triep, et al. [10]. Their design requires expensive and complicated micro-fabrication. On the other hand, our primary interest is on how to implement the theory of oscillatory, shear-driven, fluid flow for a design of a practical inexpensive micro-fluidic pump for blood flow and other applications. A harmonic excitation Stokes' $2^{\text {nd }}$ problem results in a zero net flow for Newtonian fluid. A nonNewtonian fluid may experience small drift due to nonlinear coupling between shear rate, shear stress and viscosity. Using harmonic wall excitations basically is useless for any practical pump design as the fluid only oscillates back and forth. Therefore, in our study we use oscillatory non-harmonic excitation of one or more channel walls. This approach resulted in a positive flow rate and practical design of a shear-driven micro-fluidic pump. Simple mechanisms can be designed to induce various periodic, yet non-harmonic oscillations. A Fourier analysis is used to decompose such periodic motion in various harmonics.

We are solving nonlinear diffusion-type oscillatory boundary-layer equation for periodic vibrations on one or both side-walls to simulate our 2D micro-fluidic pump. The shear stress produced by oscillating flow generates heat which is accounted for as a Rayleigh dissipationfunction heat source in the thermal-energy diffusion equation. Conservative explicit and implicit Crank-Nicolson finite-difference methods [11-15] are used to solve the differential conservation equations for mass, linear momentum, and energy. Here, we only present simple Power-Law shear-thinning model of blood flow. The results of other blood-models, 
such as Casson or Quamada $[9,16]$ will be shown elsewhere. Besides numerical efforts, many closed-form analytical solutions (not shown here) have been obtained for the Newtonian fluids and periodic boundary conditions using Eigenfunction expansion, Laplace and Fourier integral transform, and Duhamel's principle. We used Fourier decomposition of the ramp function governing the wall motion. Using the same Fourier methodology various oscillatory boundary conditions (BC) could be applied to obtain various flow patterns and positive flow rates.

\section{Mathematical Model:}

We are starting from the general, time-dependent, compressible, 2D, boundary-layer (BL) equations $[2,17]$, describing mass, linear momentum and energy conservation:

$\frac{\partial \rho}{\partial t}+\frac{\partial(\rho u)}{\partial x}+\frac{\partial(\rho v)}{\partial y}=0 \quad p=p(\rho, T) \quad \mu=\mu(T)$

x Motion : $\rho\left(\frac{\partial u}{\partial t}+u \frac{\partial u}{\partial x}+v \frac{\partial u}{\partial y}\right)=-\frac{\partial p}{\partial x}+\frac{\partial}{\partial y}\left(\mu \frac{\partial u}{\partial y}\right) \quad$ y Motion : $\frac{\partial p}{\partial y}=0$

$\rho c_{p}\left(\frac{\partial T}{\partial t}+u \frac{\partial T}{\partial x}+v \frac{\partial T}{\partial y}\right)=\frac{\partial}{\partial y}\left(k \frac{\partial T}{\partial y}\right)+\mu\left(\frac{\partial u}{\partial y}\right)^{2}+\beta T\left(\frac{\partial p}{\partial t}+u \frac{\partial p}{\partial x}\right) \quad \beta=-\frac{1}{\rho}\left(\frac{\partial \rho}{\partial T}\right)_{p}$

In addition, we need to specify the equation-of-state, which for incompressible liquid (blood) will simply imply constant density. We neglected body forces. Dynamic viscosity for a general non-Newtonian fluid can be a function of temperature. Appropriate BC and IC have to be specified for a given flow geometry. However, the mathematical model can be significantly simplified by assuming incompressible 1-D flow $(v=0)$, small Reynolds number (convective terms negligible) and the pressure gradient is zero; $\partial p / \partial x_{i}=0$. In this case, the nonlinear coupled model of incompressible oscillatory flow controlled by diffusion of viscosity and thermal diffusivity becomes:

$\rho \frac{\partial u}{\partial t}=\frac{\partial}{\partial y}\left(\mu \frac{\partial u}{\partial y}\right)$

$\rho c_{p} \frac{\partial T}{\partial t}=\frac{\partial}{\partial y}\left(k \frac{\partial T}{\partial y}\right)+\mu \Phi \quad \Phi=\left(\frac{\partial u}{\partial y}\right)^{2}$

$B C s: \quad u(0, t)=f_{1}(t) \quad u(h, t)=f_{2}(t) \quad T(0, t)=T_{o}$

ICs: $u(y, 0)=0 \quad T(y, 0)=T_{\infty}$

The Dirichlet BCs are given for the velocity and temperature at finite and one infinite boundary; $h \rightarrow \infty$. In the case of one semi-infinite domain with one plate oscillating, we obtain the celebrated Stokes $2^{\text {nd }}$ problem. If the other plate is located at a finite, but small distance, $h$, and is moving with the constant speed, starting from the rest, while the lower plate is immovable, we arrive to the well-known Couette flow. Our mathematical model is capable of dealing with the several limiting cases of the general problem: stationary, harmonic, and general periodic oscillations of one or both plates (vibrating walls), as well as, use of 
Newtonian or non-Newtonian fluids.

The Rayleigh dissipation function $\Phi=\sigma_{i j}^{\prime}\left(\partial u_{i} / \partial x_{j}\right)$ describes the rate at which the deviatoric stresses do irreversible work in the fluid. This work then appears as a volumetric heat source in the energy equation. The two-sided coupling between momentum and energy equation could be obtained by demanding that the dynamic viscosity is a function of temperature.

In the case of Newtonian fluid, the shear stress is a linear function of the velocity gradient:

$$
\tau=\mu\left(\frac{\partial u}{\partial y}\right) \quad \tau_{w}=\mu\left(\frac{\partial u}{\partial y}\right)_{y=0}
$$

We are using power-law or Ostwald-de Waele [9,16-17] rheological model for shear-thinning non-Newtonian blood model only. In this model, the dynamic viscosity is a function of the generalized shear rate:

$$
\mu(\dot{\gamma})=K|\dot{\gamma}|^{n-1} \quad \tau_{y x}=K|\dot{\gamma}|^{n}=K \cdot\left|\frac{d u}{d y}\right|^{n-1} \frac{d u}{d y} \quad\left(\dot{\gamma}=\frac{d u}{d y}\right) \quad \dot{\gamma}_{i j}=\frac{\partial u_{i}}{\partial x_{j}}+\frac{\partial u_{j}}{\partial x_{i}}
$$

Here, $K$ is the consistency coefficient and $n$ is the power-law index assumed to be constant over the total range. Obviously, for $n<1$, we will have shear-thinning or pseudo-plastic behavior (soft-spring effect), while for $n>1$, the fluid will show shear-thickening or dilatantfluid behavior (hard-spring effect). For, $n=1$, the fluid is pure Newtonian in which the dynamic viscosity is independent of the shear rate, while the shear stress is linearly proportional to the shear rate (velocity gradient).

We have mentioned earlier that the Dirichlet BCs could be or zero, constant, harmonic, or general periodic function. One of the main features and contributions of this work is the introduction of the ramp BCs on one or both plates. As is well known from the classical Stokes $2^{\text {nd }}$ problem and the Kelvin's solution of penetration of the temperature waves into soil [4], the lower disturbance frequencies will affect the oscillatory flow to a higher depth than the higher frequencies which dissipate in shorter distance. In addition, a pure harmonic motion (sine or cosine) really does not induce any mean motion other than for a small drift that could exist due to the nonlinear fluid properties. The unity ramp function for the plate excitation velocity is sketched in Fig. 1. The Fourier expansion of the ramp function is:

$$
f(t)=\pi-2 \sum_{k=1}^{\infty} \frac{\sin (k t)}{k} \quad f(t)=t \quad 0<t<2 \pi
$$

The plate acceleration, $b$, in a generalized ramp function $f(t)=b t$ is constant (slope of the uphill straight line) and accordingly the velocity is a linear function of time. The distance covered during one period is simply, $s=b T^{2} / 2$. 

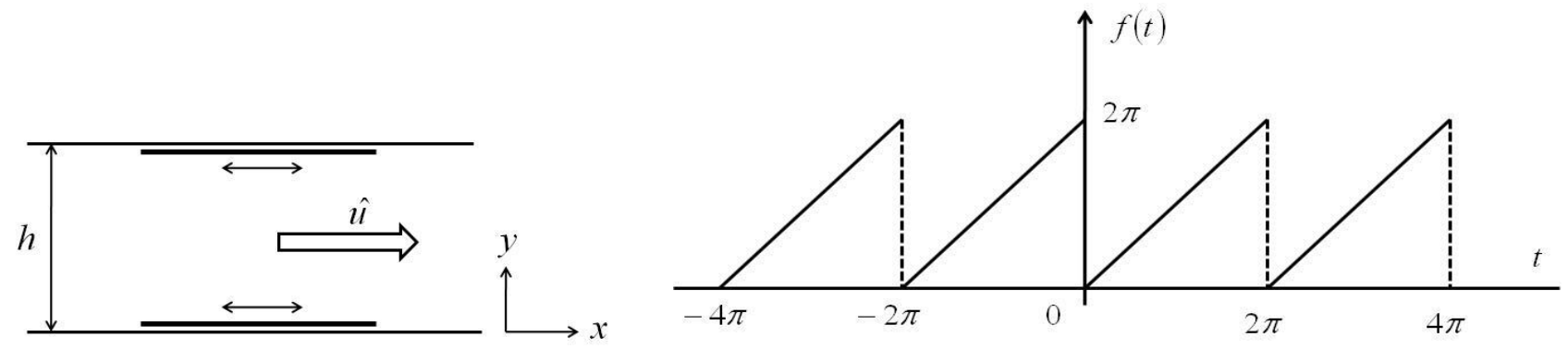

Figure (1): $2 D$ channel with oscillating wall plates and graph of ramp excitation

\section{Numerical Method:}

A forward time central space (FTCS), yet stable, explicit (Euler) finite-difference discretization scheme was used to calculate nonlinear and coupled velocity and temperature distribution in finite and semi-infinite flow domains [11-15]. Although not unconditionally stable as implicit methods, the ease of programming and execution on today's fast computers was the deciding factor. We are not showing implicit fully-conservative control-volume approach here. The discretization equations for explicit velocity and temperature fields are thus given here as:

$$
\begin{array}{ll}
u_{j}^{m+1}=u_{j}^{m}+\frac{K}{\rho_{o}} \cdot \frac{\Delta t}{(\Delta y)^{n+1}}\left[\left(u_{j+1}^{m}-u_{j}^{m}\right)^{n}-\left(u_{j}^{m}-u_{j-1}^{m}\right)^{n}\right] & \frac{K}{\rho_{o}} \cdot \frac{\Delta t}{(\Delta y)^{n+1}} \leq \frac{1}{2} \\
T_{j}^{m+1}=T_{j}^{m}+\frac{a \cdot \Delta t}{(\Delta y)^{2}}\left[T_{j+1}^{m}-2 T_{j}^{m}+T_{j-1}^{m}\right]+\frac{\mu \cdot \Delta t}{\rho \cdot c_{p}} \cdot \Phi_{j}^{m} & \Phi_{j}^{m}=\left[\left(\frac{\partial u}{\partial y}\right)_{j}^{m}\right]^{2}=\left(\frac{u_{j}^{m}-u_{j-1}^{m}}{\Delta y}\right)^{2}
\end{array}
$$

Note that the index $n$ stands for the power-law index, while $m$ is time step index. Also, if we set index $n=1$ in Eq. (5), we obtain the classical FTCS conditionally stable Euler scheme. We assumed that the dynamic viscosity is not a function of temperature for this small temperature range and that fluid conductivity and temperature diffusivity $a$ also stays constant over the same range.

\section{Results and Discussion:}

A velocity distribution in a semi-infinite geometry for cosine harmonic oscillation of one wall boundary and Newtonian fluid is shown in Fig. 2. One period $T$ last 2 seconds ( $f=0.5 \mathrm{~Hz}$ ) and basically all transients die out by then. The thickness of the Stokes' penetration layer is about $4 \mathrm{~mm}$ while the maximum velocity is $0.01 \mathrm{~m} / \mathrm{s}$. In Fig. 3, we show the velocity distribution of the steady-state oscillations of Newtonian Fluid in semi-infinite geometry with the same conditions as before. As was to be expected the average flow rate or velocity is zero averaged over one period and hence useless for any pump design. This result also agrees well with the results reported in Ai and Vafai [9]. Since we are developing practical design it is not allowed for the oscillating plate to move with high speed and simultaneously low frequency (to increase penetration depth). Such a configuration would result in unacceptably long distance of the plate movement which is not possible in a small MEMS 
design. We basically were restricting the linear movement of the plate to the order of magnitude of the channel thickness. Consequently, such pump is viable for micro-fluidic applications.

In Fig. 4, we show side-by-side velocity distributions in a finite 2D planar channel with thicknesses of $5 \mathrm{~mm}$ for a Newtonian and non-Newtonian (power-law) blood models. One wall is vibrating with the perfect ramp oscillation while the other has no-slip zero boundary value. This arrangement can be regarded as an oscillatory Couette flow. Obviously, the flow is positive averaged over one cycle and due to the nature of the ideal ramp oscillation there is never back-flow. In a practical design it is not possible to obtain a perfect ramp-excitation, so little back-flow is inevitable, but due to high frequency of the plate reverse motion, this disturbance will not propagate very far in the fluid. This is also the main idea behind our micro-fluidic pump design. In addition, one can observe that power-law blood model ( $n=0.7755$ ) [9] shows higher positive flow rates than the corresponding Newtonian model.

In Fig. 5, we show the temperature distribution in a thin 2D channel $h=5 \mathrm{~mm}$ thick with the initial temperature of $273 \mathrm{~K}$ and a fixed Dirichlet boundary temperature of $293 \mathrm{~K}$. In Fig. 6, we show side-by-side velocity distribution in a thin 2D channel, $5 \mathrm{~mm}$ thick, with both walls being excited with ramp oscillations with zero phase difference for Newtonian and non-Newtonian blood-fluid models. Again, due to the nonlinear nature of the shear rate, shear stress, and the dynamic viscosity, the flow rate of the non-Newtonian fluid is higher and more uniform over the entire cross section than Newtonian.

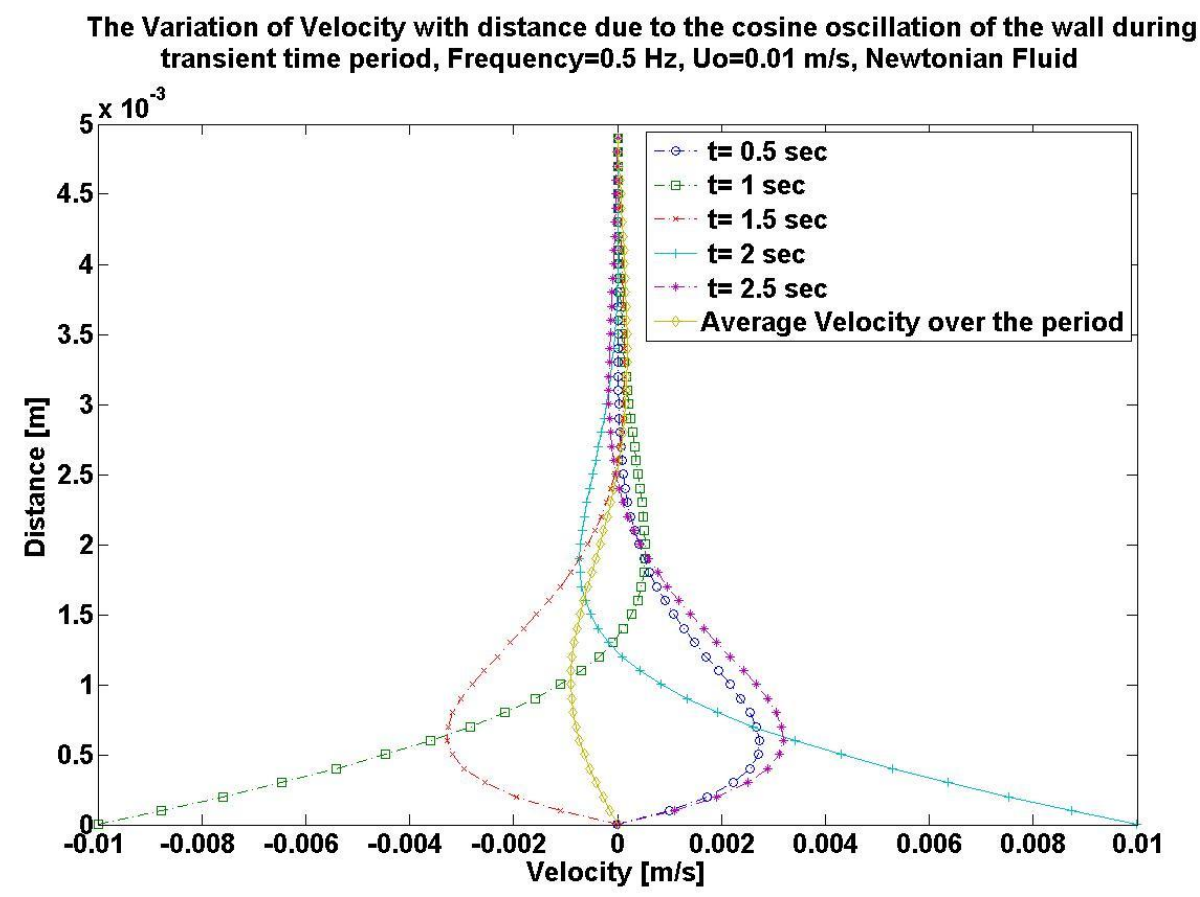

Figure (2): Transient oscillatory flow of Newtonian fluid under cosine harmonic oscillation of one wall in semi-infinite region. 


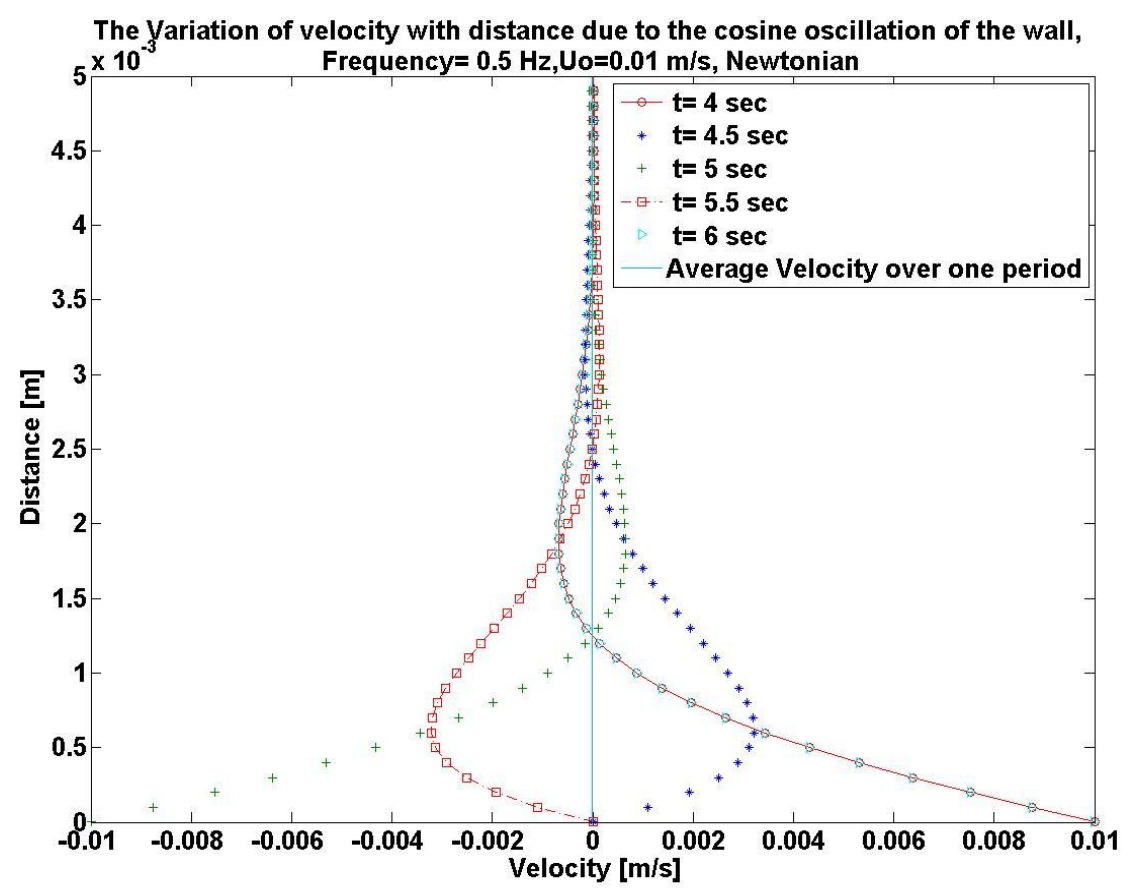

Figure (3): Developed steady-state oscillatory flow of Newtonian fluid under cosine harmonic oscillation of one wall in semi-infinite region.
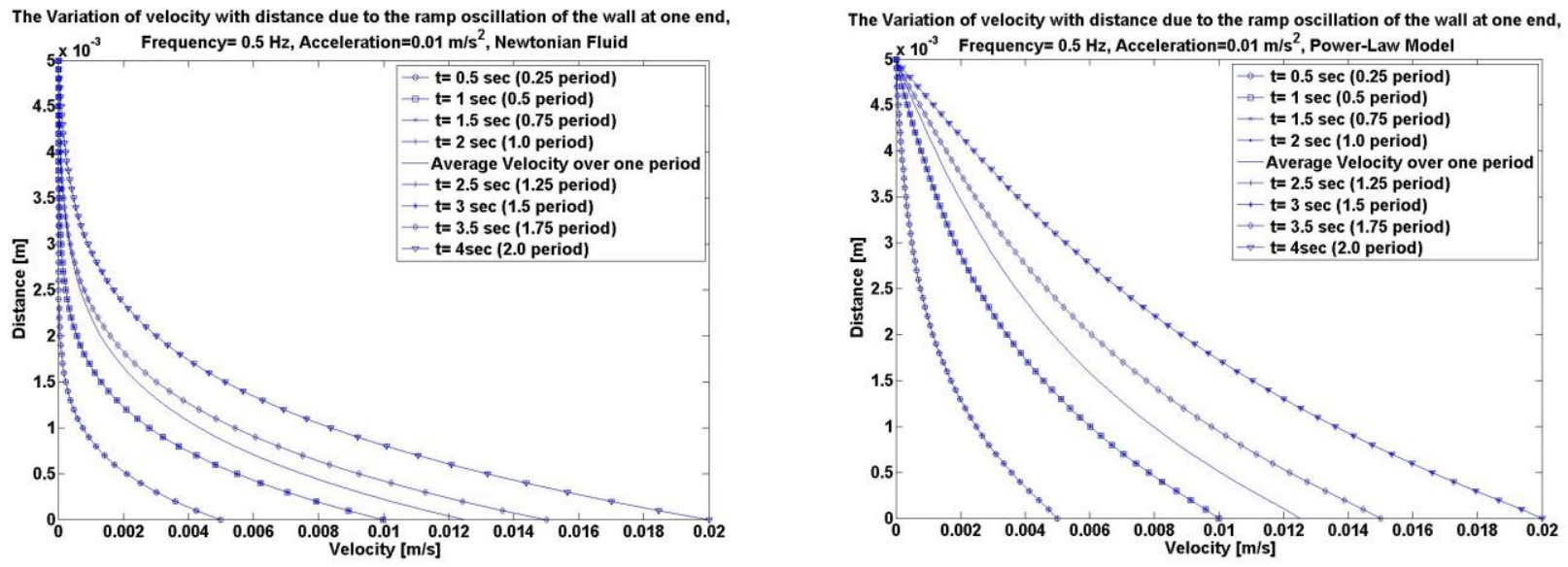

Figure (4): Velocity distribution in thin $2 D$ channel Newtonian (RHS) and non-Newtonian power-law blood-model (LHS) with the ramp oscillation of a single wall. 


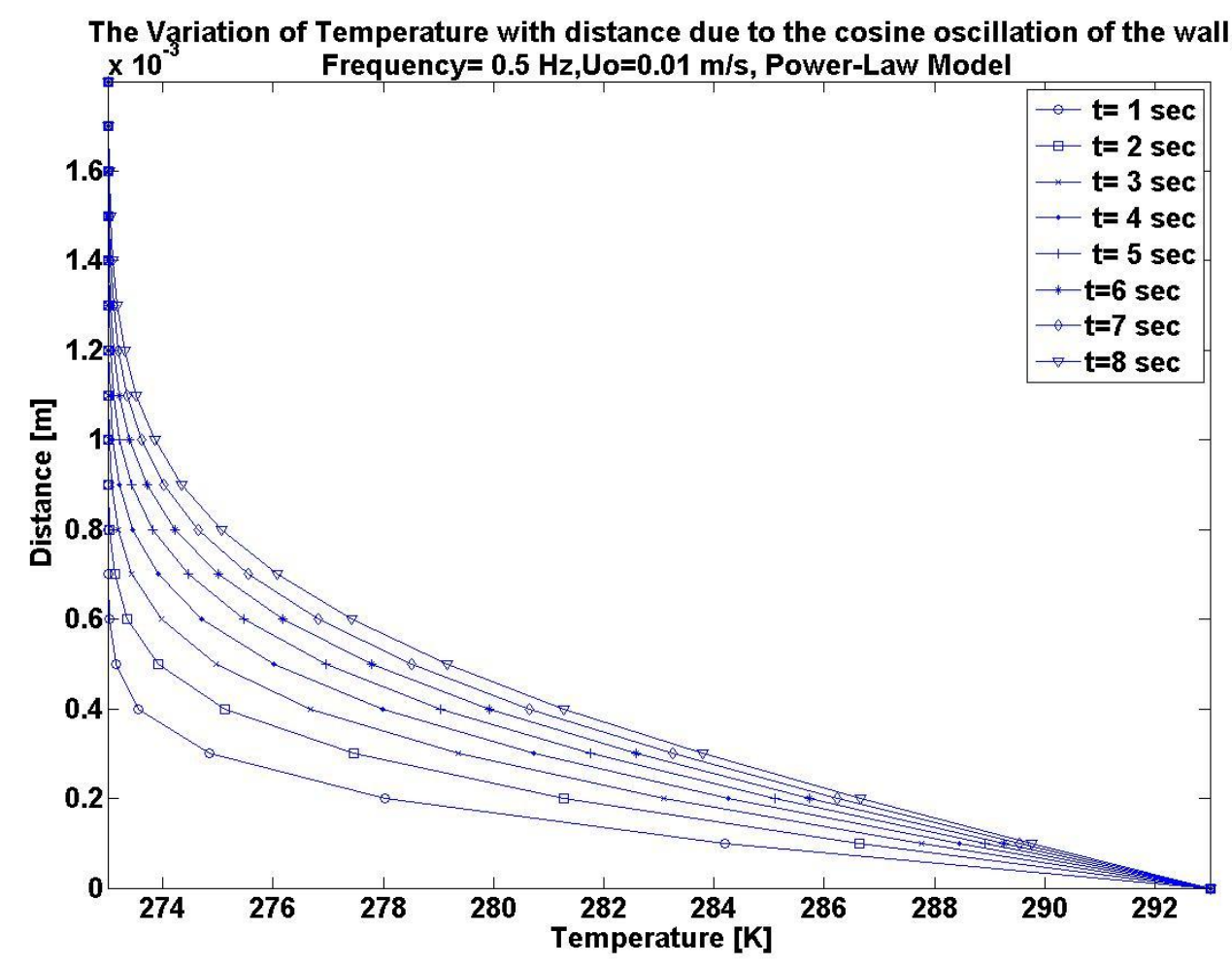

Figure (5): Temperature distribution in a $2 D$ channel for non-Newtonian blood mode and given Dirichlet Temperature and harmonic velocity boundary conditions on one wall.
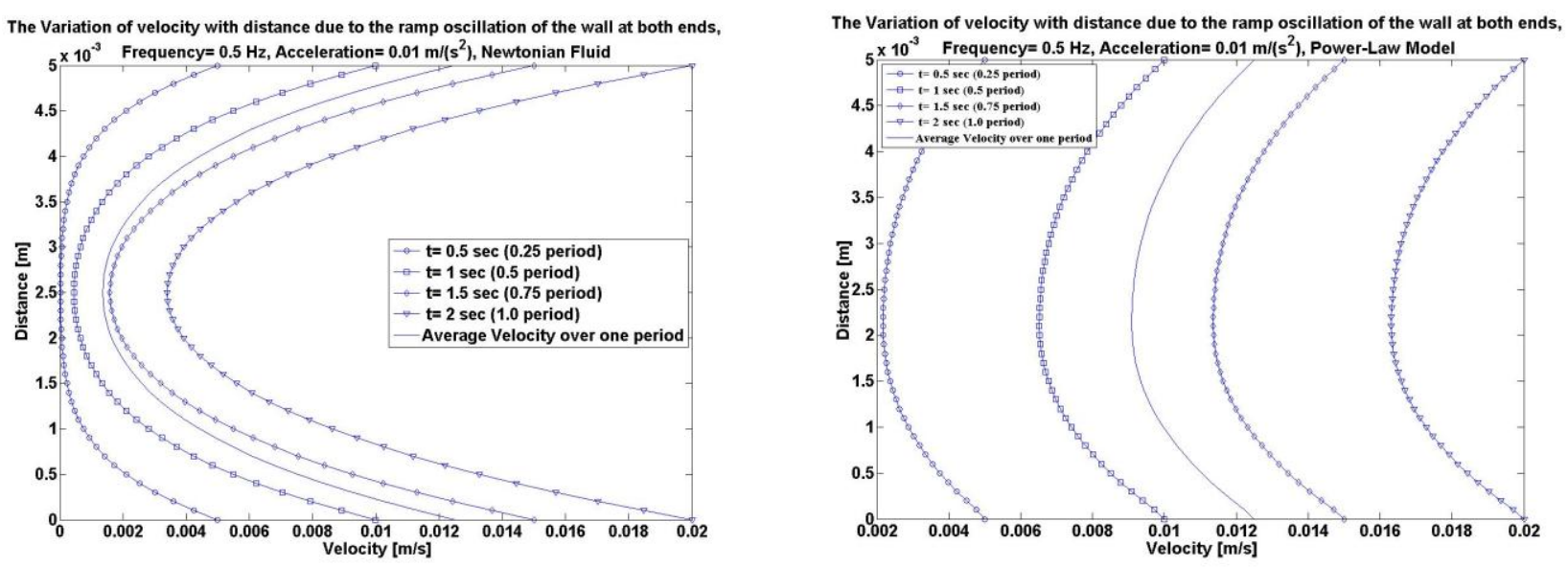

Figure (6): Velocity distribution in a $5 \mathrm{~mm} 2 D$ channel for Newtonian (RHS) and nonNewtonian (LHS) blood model and ramp boundary conditions on both walls.

\section{Conclusions:}

Due to its simple design and ease of micro-fabrication, the new shear-driven micro-fluidic pump could be used as a blood pump in cardiac support, facilitating and enabling blood flow in various, mostly small, vascular channels, etc. Shear stresses should be well below the 
critical limits for blood. One can envision applications of such miniature pump for various other bio-medical applications, such as moving fluid in a human articular cartilage, etc. The proposed micro-pump could also be used in automotive industry to help start and move highviscous oils and other fluids in low temperatures. Also, food-processing industry could see benefits from its applications. A proposed pump is ideally used in micro-fluidic MEMS systems. The non-periodic wall oscillations delivers positive flow rate whether the fluid is Newtonian or non-Newtonian and unlike pure harmonic excitation which is only "wind-milling" the fluid. Other non-periodic wall excitation and blood rheological models will be used as well. Experimental techniques, such as, PIV, LDV, MRI and/or Doppler ultrasound will be used to measure velocity profiles and verify the fidelity of the mathematical model.

\section{References:}

[1] Lamb, H., "Hydrodynamics", 6 $6^{\text {th }}$ Edition, Dover Publications, New York, 1945.

[2] Schlichting, H., "Boundary-Layer Theory", $7^{\text {th }}$ Edition (English translation by Kestin J.), McGraw-Hill, 1979.

[3] Batchelor, G.K., "An Introduction to Fluid Dynamics", Cambridge University Press, Cambridge, 2000.

[4] Carslaw, H.S., Jaeger, J.C., "Conduction of Heat in Solids", $2^{\text {nd }}$ edition, Clarendon Press, Oxford, 1959.

[5] Panton R. (1968) “The transient for Stokes's oscillating plate: a solution in terms of tabulated functions", J. Fluid Mech., Vol. 31, part 4, pp. 819-825.

[6] Erdogan, E.M. (2000) "A note on an unsteady flow of viscous fluid due to an oscillating plane wall", Int. J. Non-Linear Mechanics, Vol. 35, pp. 1-6.

[7] Khaled, A.-R.A., Vafai, K. (2004) "The effect of the slip condition on Stokes and Couette flows due to an oscillating wall: exact solutions", Int. J. Non-Linear Mechanics, Vol. 39, pp. 795-809.

[8] Muzychka, Y.S., Yovanovich, M.M., (2006) “Unsteady Viscous Flows and Stokes's First Problem", IMECE 2006-14301, pp. 1-10.

[9] Ai, L., Vafai, K., (2005) “An investigation of Stokes' second problem for non-Newtonian fluids", Numerical Heat Transfer, Part A, Vol. 47, pp. 955-980.

[10] Triep, M., Bruecker, C., Siess, T. (2006) "Numerical Simulation of the Flow in a MicroAxial Blood Pump", PAMM Proc. Appl. Math. Mech., Vol. 6, pp. 145-146.

[11] Ferziger, J.H., Perić, M., "Computational Methods for Fluid Dynamics", $3^{\text {rd }}$ Edition, ISBN 3-540-42074-6, Springer-Verlag, Berlin, 2002.

[12] Majumdar, P., "Computational Methods for Heat and Mass Transfer", Taylor \& Francis, ISBN-10: 1-56032-994-7, ISBN-13: 978-1-56032-994-7, New York, 2005.

[13] Patankar, S.V., "Numerical Heat Transfer and Fluid Flow", Hemisphere Publishing Corporation, McGraw-Hill Book Company, Washington, 1980.

[14] Press, W.H., Vetterling, W.T., Teukolsky, S.A., Flannery, B.P., "Numerical Recipes in FORTRAN: The Art of Scientific Computing", $2^{\text {nd }}$ Edition, ISBN 0-521-43064-X, Cambridge University Press, Cambridge, 1992.

[15] Thomas, J.W., "Numerical Partial Differential Equations: Finite Difference Methods", Springer-Verlag, New York, 1995.

[16] Skelland, A.H.P., "Non-Newtonian Flow and Heat Transfer", John Wiley and Sons, Inc., New York, 1967.

[17] Burmeister, L.C., Convective Heat Transfer, $2^{\text {nd }}$ Edition, John Wiley and Sons, Inc., New 
York, 1993.

\section{Nomenclatures:}

$a \quad$ Thermal diffusivity of the fluid $\left[\mathrm{m}^{2} / \mathrm{s}\right]$

$b \quad$ Constant acceleration of the wall plate $(\mathrm{s})\left[\mathrm{m} / \mathrm{s}^{2}\right]$

$\beta \quad$ Coefficient of thermal expansion $\left[\mathrm{K}^{-1}\right]$

$c_{p} \quad$ Specific heat capacity at constant pressure $\left[\mathrm{J} \mathrm{kg}^{-1} \mathrm{~K}^{-1}\right]$

$\dot{\gamma} \quad$ Shear rate $\left[\mathrm{s}^{-1}\right]$

$f \quad$ Natural Frequency of plate oscillation $[\mathrm{Hz}]$

$\Phi \quad$ Rayleigh's dissipation function $\left[\mathrm{m}^{2} / \mathrm{s}^{2}\right]$

$h \quad$ Channel height [m]

$k \quad$ Thermal conductivity [ $\mathrm{W} \mathrm{m}^{-1} \mathrm{~K}^{-1}$ ]

$K \quad$ Consistency coefficient in power-law model [Pa ${ }^{n}$ ]

$\mu \quad$ Dynamic viscosity [Pa s]

$n \quad$ Power-Law index [-]

$p \quad$ Normal Stress - pressure $[\mathrm{Pa}]$

$\rho \quad$ Density $\left[\mathrm{kg} / \mathrm{m}^{3}\right]$

$t \quad$ Time [s]

$T \quad$ Period of oscillations [s]

$T \quad$ Temperature [K]

$T_{o} \quad$ Boundary Temperature $[\mathrm{K}]$

$T_{\infty} \quad$ Initial Temperature [K]

$\tau \quad$ Shear Stress [Pa]

$u \quad$ Velocity in $\mathrm{x}$-dimension [m/s]

$B C$ Boundary Conditions

IC Initial Conditions 